\title{
LAUWAERT Françoise (dir.), Après la catastrophe
}

Bruxelles, Civilisations. Revue internationale d'anthropologie et de sciences humaines, 2007, LVI (1-2) : 218 pages

Roberte Hamayon

\section{Q OpenEdition}

Journals

Édition électronique

URL : https://journals.openedition.org/emscat/1644

DOI : 10.4000/emscat.1644

ISSN : 2101-0013

Éditeur

Centre d'Etudes Mongoles \& Sibériennes / École Pratique des Hautes Études

\section{Référence électronique}

Roberte Hamayon, «LAUWAERT Françoise (dir.), Après la catastrophe "Études mongoles et sibériennes, centrasiatiques et tibétaines [En ligne], 40 | 2009, mis en ligne le 28 décembre 2009, consulté le 13 juillet 2021. URL : http://journals.openedition.org/emscat/1644; DOI : https://doi.org/10.4000/ emscat. 1644

Ce document a été généré automatiquement le 13 juillet 2021.

(c) Tous droits réservés 


\section{LAUWAERT Françoise (dir.), Après la catastrophe}

Bruxelles, Civilisations. Revue internationale d'anthropologie et de sciences humaines, 2007, LVI (1-2) : 218 pages

\section{Roberte Hamayon}

\section{RÉFÉRENCE}

Françoise LAUWAERT (dir.), Après la catastrophe Bruxelles, Civilisations. Revue internationale d'anthropologie et de sciences humaines, 2007, LVI (1-2) : 218 p.

1 Au thème vaste et complexe proposé sous le titre d'Après la catastrophe pour ce numéro spécial de la revue Civilisations, sept chercheurs ont répondu, apportant des études de cas en tous genres observés en tous lieux.

2 Le large éventail de perspectives envisagées en introduction par Françoise Lauwaert, à qui reviennent l'initiative et la coordination de ce volume, a permis à chaque auteur d'avoir toute liberté pour choisir et traiter «sa » catastrophe. Un mot du titre donne toutefois une orientation : " après ", et l'auteur de l'introduction fait bien d'y insister. Ceci suffit à relativiser celui de "catastrophe», qui sera utilisé par l'ensemble des contributeurs non dans son sens de dénouement tragique semant la ruine et la mort, mais dans celui, dénué de connotation funeste, de «fait aux conséquences désagréables » qu'a retenu l'Académie française dans sa dernière édition. Ainsi, plusieurs des cas analysés sont plutôt des processus que des événements violents et ponctuels, et ces processus transforment plutôt qu'ils ne détruisent, si tragique la transformation soit-elle. "Après " suffit aussi à indiquer que l'accent est mis sur la temporalité d'une part - c'est des lendemains de catastrophe qu'il s'agit - sur la subjectivité d'autre part, de ceux qui les vivent comme de ceux qui les évaluent de l'extérieur. Qui en effet juge qu'il y a catastrophe, qui y croit ou n'y croit pas ? Qui la raconte, comment et à qui ? Qui en sort et comment? Tel est le questionnement que propose l'introduction, d'une écriture très foisonnante et très littéraire qui risque 
toutefois de le noyer dans une foule bigarrée d'exemples rapides (mais il « ne déplaisait pas " à l'auteur, confie-t-elle en conclusion, que "la traversée de la catastrophe se terminât dans la pénombre »). S'il se veut largement ouvert, ce questionnement reste néanmoins centré sur la reconnaissance de la catastrophe et surtout de sa mémoire, mémoire qui transforme, qui oublie, refoule ou au contraire ravive, mémoire qui instrumentalise ou réinvente, mémoire qui exacerbe une catastrophe singulière ou en superpose plusieurs.

3 C'est l'article suivant, dû à Barbara Pirlot, «Après la catastrophe. Mémoire, transmission et vérité dans les témoignages de rescapés des camps de concentration et d'extermination nazis » (pp. 21-41) qui définit en détail la problématique de la mémoire de la catastrophe, mettant en évidence certains aspects méthodologiques et théoriques importants. On sait gré à l'auteur de la clarté de sa présentation du témoignage, dont les deux composantes, le souvenir et la mise en récit, médiatisent le passé. Le témoignage est de ce fait "soumis à l'alternative du vrai et du faux ", ce qui importe d'autant plus que la portée collective de l'événement attesté est large. Mais sur quelle base peut-il être accrédité, lui que chacun est prêt à croire tout en le sachant faillible ? Suffit-il qu'il soit plausible? Encore faut-il que le témoin parvienne à exprimer son expérience (fréquent est le silence du rescapé) et que le public soit capable de comprendre cette expérience qu'il n'a pas partagée. L'histoire des témoignages sur les camps de concentration nazis montre que la fonction du témoignage a changé au fil des six décennies écoulées. Il ne s'agit plus de démontrer pour accréditer, mais de faire partager, voire d'exploiter. La diversité des usages possibles du témoignage amène l'auteur à s'interroger sur l'écriture de l'histoire et de ses liens avec la fiction et la littérature et à soulever les problèmes éthiques que peuvent parfois poser certaines exploitations artistiques des témoignages.

4 La contribution d'Isabelle Henrion-Dourcy, placée pourtant bien plus loin dans le volume, se présente comme une application d'une telle problématique de la constitution du témoignage et de son accréditation : une application vivante et subtile au cas directement observé par l'auteur. Intitulé « De l'exil à l'asile. Témoignages et authenticité culturelle des candidats tibétains au statut de réfugié politique en Belgique »(pp.121-152), l'article décrit la situation particulière de ces demandeurs d'asile qui se déclarent originaires d'un pays qui n'est pas souverain. Seuls ceux qui ont fui le Tibet au statut de province de Chine peuvent être acceptés comme tels, non ceux qui viennent de l'Inde ou le Népal où ils étaient déjà en exil, car ils ne peuvent faire état de violations des droits de l'homme (ils ne seront pas pour autant expulsés - vers la Chine? En outre, ils n'y sont pas nés). Tous doivent donc convaincre les examinateurs belges de leur authentique tibétanité. Mais comment, pour les candidats, produire des témoignages qui soient crédibles pour leurs examinateurs? Et comment, pour les examinateurs, vérifier et évaluer ces témoignages ? Il a fallu aux uns et aux autres, dit l'auteur, traverser une longue période d'ajustement mutuel. Pour les Tibétains nés au Tibet, surmonter leur réticence à se poser en victimes, à " gémir sur un malheur passé [car cela risque d']en attirer un autre »; pour ceux nés en exil, s'inventer une vie qu'ils n'ont pas vécue, dénoncer une oppression qu'ils n'ont pas subie. Et pour les examinateurs, se familiariser assez avec le mode de vie au Tibet en vue d'être capables de déceler d'où vient le candidat d'après sa réponse à des questions comme celle-ci, qui ouvre l'article : «Par quel terme désigne-t-on un yak femelle de deux ans?». Voici mis en lumière le ressort paradoxal du témoignage qui, pour être pris pour vrai, doit parfois comporter du faux, faire appel à l'invention pour restituer une réalité. Il n'est 
pas étonnant, dès lors, que les Tibétains nés en exil ressentent le refus d'asile comme un échec personnel.

5 C'est une autre potentialité du thème qu'illustrent les contributions de Laurent Legrain, Guo Yuhua et Anne-Marie Vuillemenot: les surprenants déplacements de perspective que peut révéler le point de vue de l'« après ». Les trois cas examinés ont en commun d'avoir pour cadre une situation $d^{d}$ '«après communisme " vécue par les intéressés comme douloureuse et de comporter des reconstructions mémorielles au bilan paradoxal ou ambivalent. Cet «après » est en effet ressenti comme traumatisant au point de rendre rétrospectivement positif l'état de choses auquel il a mis fin, alors qu'il était loin d'être, sur le moment, épargné pas la douleur. Mais le régime d'alors, les trois auteurs le rappellent, se livrait à un tri méticuleux de ce qui, dans le vécu de l'époque, était bon à se remémorer et possédait au plus haut degré le pouvoir d'occulter le reste. Il n'est donc pas question de catastrophe au sens événementiel du terme, et aucun des auteurs n'utilise le terme en tant que concept.

6 Pour Laurent Legrain, ce qui, chez les Darkhad de Mongolie septentrionale, parait le plus proche de l'idée de catastrophe sans l'être pour autant est la situation créée par l'« interminable transition démocratique » que connaît le pays depuis deux décennies, non pas tant cette situation par elle-même que par la reconstruction idéalisée du passé qu'elle suscite. «Au bon vieux temps de la coopérative. À propos de la nostalgie dans un district rural de la Mongolie contemporaine » (pp. 103-120) montre comment le choc de l'entrée dans le monde libéral global embellit, par contrecoup, le souvenir de celui de la collectivisation menée dans les années 1950 (la deuxième dans ce pays). L'auteur déroule une analyse fine et originale des attitudes à l'égard des lieux et des objets hérités de cette période, qui met en évidence le rôle qu'ils jouent dans la construction de la mémoire collective. L'argument le plus puissant en faveur de cette hypothèse forte de l'agentivité attribuée à des lieux et des objets du passé est aussi l'élément le plus révélateur du caractère reconstruit de la mémoire, de ce que le regard porté d'« après " réinvente et réaménage. L'un et l'autre argument tiennent à ce que cette nostalgie qui fait tant apprécier, par exemple, de se retrouver auprès du bon vieux centre culturel aux dimensions démesurées fondé en 1956 n'est pas le fait de la génération qui a souffert de cette vague de collectivisation, mais de la suivante, actuellement dans la force de l'âge, qui ne l'a pas connue. La reconstruction n'est pas pour autant une totale fabrication de la part de jeunes prêts à ne voir dans les purges que des « erreurs de jeunesse » du parti communiste. Elle se nourrit aussi de ce qu'ont dit et fait leurs aînés (bien que ceux-ci ne critiquent pas le mode de vie actuel ni ne surestiment les difficultés présentes); ce sont bien leurs actes et leurs récits qui distillent le sentiment d'abandon actuel et le regret d'un passé plus ou moins réinventé.

Par contraste, l'idéalisation d'un autre passé de collectivisation que retrace Guo Yuhua dans " 'Nous étions comme un feu ardent'. La collectivisation des esprits dans les récits des femmes du village de Jicun (nord du Shaanxi)» (pp. 43-62) est le fait même des femmes qui ont vécu le «Grand Bond en Avant» dans la Chine des années 1958-1960. Pour mettre en œuvre ce grand bond, les femmes avaient été enrôlées au même titre que les hommes. Mais alors que les hommes relatent cette période avec précision, les femmes, que l'auteur a dû, non sans mal, "amener à la parole », n'en ont de souvenirs qu'à travers leur expérience directe, surtout celle vécue dans leur propre corps: l'épuisement, les souffrances dues à la dureté des travaux, la faim, la leur et celle de leurs enfants en bas âge. Ce qui frappe l'auteur à l'écoute de ces récits de souvenirs 
chargés d'émotions si douloureuses, ce sont les éclairs de joie et d'enthousiasme qui les ponctuent à l'évocation des travaux collectifs ou des mobilisations politiques. "On chantait, on bavardait, on riait " disent ces femmes qui en ont retenu l'atmosphère égalitaire, solidaire et entraînante - pour elles une vraie découverte. Aller aux champs, fût-ce pour les travaux les plus durs, c'était sortir de chez elles, c'était lever l'oppression des pouvoirs traditionnels qui les confinaient à leurs fourneaux, privées de contacts, de partage et d'entraide, c'était pouvoir apprendre des caractères ou se faire appeler par leur nom individuel, c'était supprimer un bon nombre de différences avec les hommes. En somme, la douleur de la pénurie matérielle et la joie de la plénitude spirituelle s'entremêlent dans la mémoire de ces femmes alors que leur meilleure situation de vie paysanne dans la Chine actuelle leur donne un sentiment de marginalité. D'ailleurs, elles justifient la politique maoïste consistant à propager "l'amer puis le doux ", ce en quoi l'auteur voit à juste titre une confirmation de l'idée bourdieusienne que les dominés finissent toujours par légitimer la violence symbolique des dominants. Les récits de ces femmes montrent à quel point elles se sont senties elles-mêmes acteurs du projet auquel elles participaient.

Le titre qu'Anne-Marie Vuillemenot donne à son article (pp. 87-100) «Le jour où les mondes disparurent" est celui d'une nouvelle kazakhe qui relate une journée de printemps des années 1960. C'est l'époque où l'URSS engage au Kazakhstan des essais nucléaires à ciel ouvert, déplaçant une partie de la population pour permettre d'évaluer les effets de l'irradiation sur l'autre partie, laissée sur place. Tel est l'ancrage que l'auteur choisit pour présenter la société kazakhe traditionnelle et en particulier la vie du berger nomade, dont l'histoire va "de cataclysme en cataclysme" au fil du $\mathrm{xx}^{\mathrm{e}}$ siècle. Une vie faite de bouleversements et de ruptures qui modifient en profondeur tant la société que la famille et l'individu. Ainsi, la femme qui fait vivre les siens de l'argent que lui vaut un travail à l'extérieur se libère des contraintes de la vie domestique et prend goût à la vie urbaine. Le berger, lui, est désemparé, en quête d'une identité redéfinie dans sa société devenue sédentaire. Ceci conduit l'auteur à soulever d'intéressantes questions de portée politique et métaphysique : "comment l'individu contemporain se reconnaît-il dans la personne kazakhe traditionnelle? ", «comment l'organisation cosmogonique a-t-elle pu disparaitre alors qu'elle avait résisté à la soviétisation? ». La personne kazakhe était caractérisée par de solides liens familiaux et de riches principes vitaux. Or l'individuation a entrainé la perte des repères collectifs et le repli sur soi, alors que l'idéologie de l'Homo sovieticus avait permis de préserver dans une certaine mesure l'identité kazakhe et divers référents culturels. Les Kazakhs vont-ils «adopter nos crèches et nos mouroirs?» Constatant que le passage d'une identité locale à une identité nationale signifie se référer à une communauté imaginaire, tout comme la communauté mondiale à laquelle renvoie l'affirmation actuelle d'identité musulmane, l'auteur suggère que l'inscription dans un monde virtuel globalisé revient à transformer le " réel en sa représentation ».

9 L'article d'Olivia Angé est le seul à traiter non des mots mais des actes de l'après catastrophe. "'Ici nous troquons ton désespoir pour de l'espérance...' Approche ethnographique du troc urbain dans une société en crise » (pp. 63-85). L'auteur décrit et analyse le réseau de troc urbain qui se met en place en Argentine à la suite de la crise de 2001, à l'initiative de la classe moyenne. La crise ébranle la société tout autant sur le plan idéologique que sur le plan économique : le peuple argentin, fier de sa réputation de peuple affectueux, se découvre soudain violent. La porte s'ouvre à l'utopie et à la tentation de la rendre réelle. C'est ainsi que voit le jour le système économique 
informel imaginé par les trois pères fondateurs de l'idéal du bon " prosommateur », qui doit consommer une quantité égale à la production qu'il a écoulée. Leur projet, qui vise à montrer qu'il est possible d'être productif à partir du seul capital humain, consiste à faire d'une forme de troc sans réciprocité immédiate une voie de socialisation. Le système procède d'abord par simple notation comptable dans le carnet du club organisateur par lequel transitent les marchandises. Par la suite sont créés des bons d'échange, les credito, sur lesquels sont mentionnés les noms du débiteur et du créditeur ; ils ne doivent ni circuler entre les partenaires ni être accumulés mais remis au club organisateur, entre les mains duquel ils constituent une promesse de répétition des échanges dans le long terme. Ces bons d'échange sont peu à peu reconnus à l'échelle nationale et fonctionnent comme une sorte de monnaie. Mais la mauvaise gestion de certains clubs et les falsifications de credito finiront par entraîner la chute du système. Durant la période où il a fonctionné cependant, il a permis de restaurer des pratiques sociales de partage et d'entraide, qui ont favorisé une recomposition identitaire, tant il est vrai que, rappelle l'auteur à la suite de Simmel : ce n'est pas la monnaie qui est responsable de la dissolution des liens sociaux, mais l'usage qui en est fait.

Dans « Comme une écaille sur le mur. À propos de Le Dernier caravansérail (Odyssées), un spectacle en deux parties du Théâtre du Soleil» (pp.159-182), Françoise Lauwaert invite le lecteur à comparer au travail de l'anthropologue le travail qu'ont fait les comédiens du Théâtre du Soleil pour ce spectacle, qu'elle décrit en détail. Ceux-ci se sont rendus à Sangatte pour recueillir des récits d'immigration en vue de les recréer ensuite sur scène. Outre les questions de l'exil, du déracinement, de la reconstruction de la vie après une catastrophe qui alimentent le contenu des récits originels comme des récits recréés, l'auteur aborde la question de la forme, qui lui paraît primordiale quand il s'agit «de parler de l'autre sans trop le trahir». Si l'aspect artistique prime pour le comédien, sa tâche est, comme celle de l'anthropologue et du psychanalyste, de donner à son matériau une forme qui le rende pensable. C'est le souci de la forme qui leur permet de réaliser leur objectif commun, celui, souligne l'auteur, de " produire un discours vrai ». C'est pourquoi, ajouterais-je, il y a toujours plusieurs « discours vrais » possibles.

Deux articles hors thème terminent ce volume, tous deux solidement informatifs, l'un de Jeanne Semin «L'argent, la famille, les amies. Ethnographie contemporaine des tontines africaines en contexte migratoire » (pp. 183-199), l'autre de Eva Hejdova « La crevette et la gouvernance : les dynamiques des entreprises aquacoles dans la province de Pampanga aux Philippines (pp. 201-218).

Pour conclure ma recension de ce riche et intéressant volume, j'aimerais suggérer au lecteur non seulement - en écho à l'introduction de Françoise Lauwaert - de centrer son attention sur le premier mot du titre, « après », mais d'oublier celui de catastrophe. C'est cet « après » qui constitue un facteur de cohérence entre les contributions, alors que le terme de catastrophe y est employé de façon si disparate qu'il en perd toute spécificité. Cette disparité, ce flou conceptuel, l'absence de problématique de la catastrophe ont, je l'avoue, gêné ma propre lecture comme ils ont, me semble-t-il, gêné la rédaction de plusieurs contributeurs qui ont, comme pour compenser, multiplié les synonymes. Encore s'agit-il d'un «après » bien partiel puisqu'il ne concerne que ceux pour lesquels il existe un "après ", ceux qui ont survécu à ladite catastrophe et en tirent, d'une façon ou d'une autre, une sorte de leçon, laissant à l'écart ceux qui - il en 
existe - en tirent profit. Mais l'« après » qui sous-tend ce recueil a le grand intérêt de rendre sensible le travail du temps en tant que tel, qu'il soit le fait de la mémoire, de la réaction, de la réinvention ou de l'instrumentalisation. C'est bien l'analyse de ce travail du temps qui permet de comprendre que ce qui a été vécu dans la douleur puisse être remémoré avec plaisir, ou inversement qu'une situation ressentie comme heureuse soit rétrospectivement perçue comme désastreuse, de comprendre, en somme, des retournements de catastrophe, ainsi que pourraient être qualifiées les situations décrites par Laurent Legrain et Guo Yuhua. Et c'est bien une telle analyse du travail du temps qui peut mettre au jour les mécanismes qui donnent lieu aux réalités humaines. 